\title{
Home enteral feed dietitians save unnecessary hospital admissions of patients with percutaneous endoscopic gastrostomy (PEG)
}

\author{
S. White, G. Simpson, J. Grant, A. J. Anderson, D. S. Sanders and M. E. McAlindon \\ Sheffield Teaching Hospitals NHS Trust
}

\begin{abstract}
Having shown that PEG-related complications are a common cause of hospital readmission ${ }^{(1)}$, two home-enteral-feed (HEF) dietitians were appointed to provide patient support in the community by regular dietetic monitoring and having the ability to respond to gastrostomy-related problems efficiently to prevent hospital re-admission. They also provide training to patients, carers and other health care professionals on how to deal with tube complications including displacement. All patients were discharged with an aftercare protocol including advice and contact numbers in the event of problems.

Data for the year ending February 2007 were collected prospectively using the Infoflex PEG database.

The caseload at the year end was 180 patients. Over this period, a total of 245 individual patients were home-enterally-fed at some point. Seventy-nine of these were new patients of whom $91 \%$ were seen within 5 working days (mean $2.9 \mathrm{~d}$ ) of hospital discharge. There were 1514 follow-up contacts (home, $n$ 825; nursing or residential home, $n 392$; telephone, $n$ 303; out-patients, $n 3$ and hospital inpatients, $n$ 2). A total of 545 PEG-related complications were dealt with (over-granulated stoma, $n$ 98; broken Y adaptor, $n$ 88; broken clamp, $n$ 50; leaking stoma, $n$ 47; fixation device too tight, $n$ 23; damaged tube, $n 15$ and other, $n$ 123). A total of 101 patients required new balloon retained gastrostomies (seventy-one balloon gastrostomies and thirty low profile gastrostomies), fifty-eight as emergency procedures (following PEG displacement, tube damage or blockage) and forty-three planned, with no complications. Although sixty-nine patients were admitted during this year, only fifteen $(21 \%)$ were for PEG problems and all occurred at times of non-availability of staff at weekends or holidays or failure of carers to adhere to the written aftercare protocol.

Hospital admissions were avoided in all fifty-eight instances (thirty patients, $12 \%$ of the total cohort of 245 patients) of PEG displacement, damage or blockage by emergency replacement by the HEF dietitians. However, it is likely that the early diagnosis and treatment of less urgent complications, and the training of patients, carers and other health care professionals will have avoided many further admissions.
\end{abstract}

1. Sanders DS et al. (2001). Percutaneous Endoscopic Gastrostomy: a prospective analysis of hospital support required and complications following discharge to the community. Eur J Clin Nutr 55, 610-614. 